\title{
Is diffusion MRI the future biomarker to measure therapeutic efficacy in multiple sclerosis?
}

\author{
1,2ZZsigmond Tamas Kincses \& ${ }^{1,3}$ László Vécsei \\ ${ }^{1}$ Department of Neurology, University of Szeged \\ ${ }^{2}$ Department of Radiology, University of Szeged \\ ${ }^{3}$ MTA-SZTE Neuroscience Research Group, Szeged, Hungary
}

Corresponding author:

Zsigmond Tamás Kincses MD, PhD

Neuroimaging Research Group

Department of Neurology

Albert Szent-Györgyi Clinical Center

University of Szeged

Semmelweis u. 6

6725-Szeged

HUNGARY

e-mail: kincses.zsigmond.tamas@med.u-szeged.hu

www: www.nepsy.szote.u-szeged.hu/ kincsesz

This article has been accepted for publication and undergone full peer review but has not been through the copyediting, typesetting, pagination and proofreading process, which may lead to differences between this version and the Version of Record. Please cite this article as doi: 10.1111/ene. 13591

This article is protected by copyright. All rights reserved. 
MRI is the cornerstone of the diagnosis of multiple sclerosis (MS), and in recent years it was advocated for the follow up of the efficacy of the ever-growing number of disease modifying treatments (DMT). While in the clinical practice the most important biomarkers are still the $\mathrm{T} 2$ and T1 enhancing lesion burden, clinical studies are already utilising atrophy estimation. These measures cover a vast majority of MS pathology, namely the perivenular inflammation and gliosis in the white matter lesions as well as grey matter pathology. However, neither lesion burden nor atrophy is specific to the underlying pathology and both of these measures neglect to show the detrimental processes of the normal appearing white matter (NAWM). Quantitative MRI approaches could be of use to describe the various aspects of underlying pathological changes. In particular, various parameters estimated from diffusion tensor imaging (DTI) have been shown to correlate with demyelination and axonal loss in the white matter.

In this issue of the European Journal of Neurology, Zivadinov et al. in a prospective, observational, longitudinal study investigated various diffusion parameters in the NAWM and in the thalamus as potential biomarkers to monitor the effectivity of dimethyl-fumarate in treating relapsing remitting MS patients [1]. They acquired diffusion data at two different field strengths and in thirty noncollinear diffusion directions. There were three important results of the study: (i) At baseline, there was an extensive white matter disintegration in MS patients not only restricted to lesions, but also affecting the vast majority of the NAWM too. (ii) Over a 24-month follow-up period, there was no significant change in diffusion parameters in both healthy and MS patients alike. (iii) In a comparison of the two groups, MS patients showed slower deterioration of diffusion parameters compared to the healthy controls.

A conclusion one could draw from the first two results could be that the therapy stopped the disintegration of the white matter and also the thalamus in MS. This is indicated by the significant intergroup differences, which did not further deteriorate when patients were on treatment. However, an alternative conclusion could be that changes in the diffusion parameters occur much slower than the time allowed for the follow-up period of the study. This conclusion is however made unlikely, considering that the rate of change in diffusion parameters was different between the two groups.

This pioneering study calls attention to several outstanding questions, which need to be considered in future investigations.

This article is protected by copyright. All rights reserved. 
1. What are the appropriate parameters necessary in order to acquire diffusion data? Diffusion tensor imaging was shown to have good reproducibility over various vendors and field strengths $[2,3]$. In regard to the number of diffusion directions needed, former theoretical analyses and simulations made suggestions [4], but real life data especially of patients and in regions of specific interest for analysis (thalamus, white matter skeleton) is still sparse. Due to the duration of high diffusion direction studies, these questions are critical and the utilisation of novel techniques, such as multiband acquisitions should be investigated.

2. The effect of biological factors on brain diffusion parameters (e.g. hydration, caffeine consumption, menstrual cycle etc.) should be understood. Special attention should be given when considering the effects of drugs, for example mega-dose steroid treatment. The antiinflammatory effect of DMTs could result in similar effect as in case of brain volume, termed pseudo-atrophy. Conversely, acute relapses may also have an effect on diffusion parameters outside the lesions.

3. The natural course of the disease is not well known in terms of diffusion alterations, but the acquisition of such data is not straightforward given the increasing number of established effective therapies [5]. In large population studies, the stable and clinically (or other MRI parameter-wise) deteriorating patients should at the very least be investigated in order to learn about the magnitude of diffusion parameter changes.

4. The relationship of diffusion parameters to clinical disability and cognitive dysfunction should be analysed. While lesion load and disability has only modest correlation, known as the clinico-radiological paradox $[6,7]$, the correlation of disability with brain atrophy seems to be stronger [8]. However, the contribution of various MRI parameters (lesion load, cortical and subcortical brain atrophy, diffusion alterations etc.) to clinical and cognitive dysfunction has not been analysed systematically. Special attention needs to be paid to the possible interrelatedness of the changes in these parameters [9].

5. Finally, the outstanding question is if diffusion is a sensitive enough biomarker to enter the world of clinical trials and everyday clinical practice. In order to do this, easy to implement and easy to interpret analysis approaches, usable on a single subject level should be developed.

Zivadinov's study contributes significantly to the above listed outstanding issues, may trigger further research and have implications for the design of future clinical trials.

This article is protected by copyright. All rights reserved. 


\section{Disclosure of conflicts of interest}

The authors declare no financial or other conflicts of interest.

[1]. Zivadinov R, Hagemeier J, Bergsland N, Tavazzi E, Weinstock-Guttman B. Effect of dimethyl fumarate on gray and white matter pathology in subjects with relapsing multiple sclerosis: a longitudinal study. European Journal of Neurology. 2018.

[2]. Palacios EM, Martin AJ, Boss MA, et al. Toward Precision and Reproducibility of Diffusion Tensor Imaging: A Multicenter Diffusion Phantom and Traveling Volunteer Study. AJNR Am J Neuroradiol. 2017 38: 537-545.

[3]. Grech-Sollars M, Hales PW, Miyazaki K, et al. Multi-centre reproducibility of diffusion MRI parameters for clinical sequences in the brain. NMR Biomed. 2015 28: 468-485.

[4]. Chen Y, Tymofiyeva O, Hess CP, Xu D. Effects of rejecting diffusion directions on tensorderived parameters. Neuroimage. 2015 109: 160-170.

[5]. Polman $\mathrm{CH}$, Reingold SC, Barkhof $\mathrm{F}$, et al. Ethics of placebo-controlled clinical trials in multiple sclerosis: a reassessment. Neurology. 2008 70: 1134-1140.

[6]. Barkhof F. The clinico-radiological paradox in multiple sclerosis revisited. Curr Opin Neurol. 2002 15: 239-245.

[7]. Kincses ZT, Ropele $S$, Jenkinson $M$, et al. Lesion probability mapping to explain clinical deficits and cognitive performance in multiple sclerosis. Mult Scler. 2011 17: 681-689.

[8]. Kincses ZT, Toth E, Banko N, et al. Grey matter atrophy in patients suffering from multiple sclerosis. Ideggyogy Sz. 2014 67: 293-300.

[9]. Toth E, Szabo N, Csete G, et al. Gray Matter Atrophy Is Primarily Related to Demyelination of Lesions in Multiple Sclerosis: A Diffusion Tensor Imaging MRI Study. Front Neuroanat. 2017 11: 23.

This article is protected by copyright. All rights reserved. 